\title{
Strong Optical-Mechanical Coupling in a Vertical GaAs/AIAs Microcavity for Subterahertz Phonons and Near-Infrared Light
}

\author{
A. Fainstein, ${ }^{1}$ N. D. Lanzillotti-Kimura, ${ }^{1}$ B. Jusserand, ${ }^{2}$ and B. Perrin ${ }^{2}$ \\ ${ }^{1}$ Instituto Balseiro and Centro Atómico Bariloche, C.N.E.A., R8402AGP Bariloche, Río Negro, Argentina \\ ${ }^{2}$ Institut des Nanosciences de Paris, UMR 7588 C.N.R.S., UPMC, 75005 Paris, France
}

(Received 6 April 2012; published 14 January 2013)

\begin{abstract}
We show that distributed Bragg reflector GaAs/AlAs vertical cavities designed to confine photons are automatically optimal to confine phonons of the same wavelength, strongly enhancing their interaction. We study the impulsive generation of intense coherent and monochromatic acoustic phonons by following the time evolution of the elastic strain in picosecond-laser experiments. Efficient optical detection is assured by the strong phonon backaction on the high- $Q$ optical cavity mode. Large optomechanical factors are reported $(\sim \mathrm{THz} / \mathrm{nm}$ range). Pillar cavities based in these structures are predicted to display picogram effective masses, almost perfect sound extraction, and threshold powers for the stimulated emission of phonons in the range $\mu \mathrm{W}-\mathrm{mW}$, opening the way for the demonstration of phonon "lasing" by parametric instability in these devices.
\end{abstract}

PACS numbers: 78.20.Pa, 42.50.Wk, 78.47.J-, 78.66.Fd

The interaction of optical and mechanical resonances in cavities has attracted strong interest motivated by a myriad of new phenomena and applications [1-13]. Sound amplification by stimulated emission (the "saser") has also been sought since the development of the laser itself [14,15] with light-sound cavities being early understood as a means to accomplish this goal [16-20]. Optoacoustic parametric oscillatory instabilities in Fabry-Perot light-sound resonators [18] have been proposed to induce positive gain with acoustic signal amplification [17-20]. In these devices, light is coupled through an optical cavity mode and is confined within the resonator walls where it excites acoustic modes of the resonator. The cavity is modulated at the vibrational frequencies leading to strong backaction on the light field. These effects have been mostly studied at $\mathrm{MHz}-\mathrm{GHz}$ frequencies in microspheres, toroidal microcavities, membranes, MEMS, and planar photonic waveguides $[21,22]$. Such microsystems are attractive because they can attain high optical and acoustic $Q$ factors (up to $10^{7}$ ), with the implication of very strong coupling even at low laser powers. Concomitant with this high finesse, however, results in a rather poor extraction of both photons and vibrations out of the device.

Here we propose a new and different approach to cavity optomechanics. We report strong optomechanical coupling at the sub- $\mathrm{THz}$ acoustic vibration range in planar III-V semiconductor monolithic multilayer cavities. These devices are based on the structures developed to demonstrate single-photon emitters [23] and vertical cavity surface emitting lasers (VCSELs) [24]. We show that for the GaAs/AlAs family of materials, a resonator structure based on distributed Bragg reflectors (DBRs) and designed to confine photons (i.e., an optical microcavity) efficiently confines acoustic phonons of the same wavelength. These structures exploit the unsurpassed growth quality of molecular beam epitaxy, and as we will demonstrate, constitute optomechanical devices that can attain very high mechanical and optical $Q$ factors $\left(Q \sim 10^{5}\right)$, very low mechanical effective masses $\left(m_{\mathrm{eff}} \sim \mathrm{pg}\right)$, large optomechanical coupling factors $\left(g_{\mathrm{om}} \sim \mathrm{THz} / \mathrm{nm}\right)$, and ultrahigh vibrational frequencies (sub-THz), while displaying at the same time very efficient extraction of both photons and phonons.

Optical and acoustic DBRs are periodic sequences of bilayers with contrasting physical properties: either optical (index of refraction $n_{i}$, and hence light speed $c_{i}$ ) or acoustic (sound speed $v_{i}$ and material density $\rho_{i}$ ). The optimal relation of thicknesses between the two materials is determined by the light and sound speeds ratio. That is, $d_{1} / c_{1}=$ $d_{2} / c_{2}=1 / 4 \nu_{\mathrm{opt}}$ and $d_{1} / v_{1}=d_{2} / v_{2}=1 / 4 \nu_{\mathrm{ac}}$, respectively [25]. Here $\nu_{\mathrm{opt}}$ and $\nu_{\mathrm{ac}}$ are the optical and acoustic frequencies. The reflectivity of such a $\lambda / 4$ DBR mirror $R \approx 1-4 Z^{2 N}$ is defined by the number of bilayer periods $N$ and the impedance mismatch $Z$. The latter is $Z_{\text {op }}=$ $c_{1} / c_{2}$ or $Z_{\mathrm{ac}}=\rho_{2} v_{2} / \rho_{1} v_{1}$ for the optical or acoustic case, respectively. A resonant cavity is obtained by growing two DBRs enclosing a spacer. It is the reflectivity $R$, and hence the impedance mismatch $Z$, that determines the $Q$ factor of a cavity. The optimal spacer thickness corresponds to an integer number $p$ of half wavelengths, and is given by $d_{\mathrm{cav}}=p c_{\mathrm{cav}} / 2 \nu_{\mathrm{opt}}$ with $c_{\mathrm{cav}}$ the light velocity in the spacer material. "Optimal" implies that the cavity mode falls at the center of a DBR stop band, and the $Q$ factor is the largest for the materials chosen [26]. Typically, DBR semiconductor cavities have $Q$ factors $\sim 10^{3}-10^{5}$ depending on $N$ [27]. The key and new concept here is that for GaAs (material 1) and AlAs (material 2) a "double magic" coincidence exists: 1) the ratios of (light and sound) velocities are almost identical $\left(c_{2} / c_{1} \sim v_{2} / v_{1} \sim 1.2\right)$ 
and 2) the (light and sound) impedance contrasts are also almost identical $\left(Z_{\mathrm{opt}} \sim Z_{\mathrm{ac}} \sim 0.83\right)$. This implies that the same optical microcavity optimized to confine photons will optimally confine acoustic phonons of the same wavelength and with the same $Q$ factor [25].

We consider a $\lambda / 2$ GaAs-spacer vertical microcavity with DBRs made of $\mathrm{Al}_{0.18} \mathrm{Ga}_{0.82} \mathrm{As} / \mathrm{AlAs}$ layers (see scheme in Fig. 1), 20 pairs on the bottom, 18 on top. The simultaneous optimization of the light and sound cavity is illustrated in Figs. 1(b)-1(d): both narrow optical and phonon cavity modes are well centered within the corresponding reflectivity stop bands $\left(Q \sim 10^{3}\right.$ for both light and sound), and both resonant optical intensity and square displacement are strongly amplified and precisely overlap at the cavity spacer.

In a typical cavity optomechanics experiment, the wavelength of a cw laser is tuned to the flank of the cavity optical resonance $[21,22]$. The cavity vibrations modulate the optical transmission, and with a spectrum analyzer vibrational spectroscopy is performed. The change in transmission can be expressed as $\Delta T=\left(\frac{d T}{d \omega}\right)\left(\frac{d \omega}{d \alpha}\right) \Delta \alpha$. Here $\frac{d T}{d \omega}$ is the derivative of the cavity mode transmission peak (a function of the cavity $Q$ factor), and $\Delta \alpha$ is the maximum displacement of the involved mechanical mode $[10,22]$. The magnitude of the light-sound interaction can be quantified by the optomechanical coupling factor $g_{\text {om }}=$ $d \omega / d \alpha$ which describes the variation of the cavity mode angular frequency $\omega$ with the mechanical displacement $\alpha$ [10]. Based on the expression for $d_{\text {cav }}$ given above, we write for the angular frequency $\omega=\pi p c / n_{\text {cav }} d_{\text {cav }}$, with $n_{\text {cav }}$ the spacer refractive index. By identifying $\Delta \alpha$ with the phonon induced variation in cavity length $d_{\text {cav }}$, we obtain as a first order approximation $g_{\text {om }}=-\omega / d_{\text {cav }}$. For a $\lambda / 2$ microcavity with $\omega$ resonantly tuned to the GaAs gap
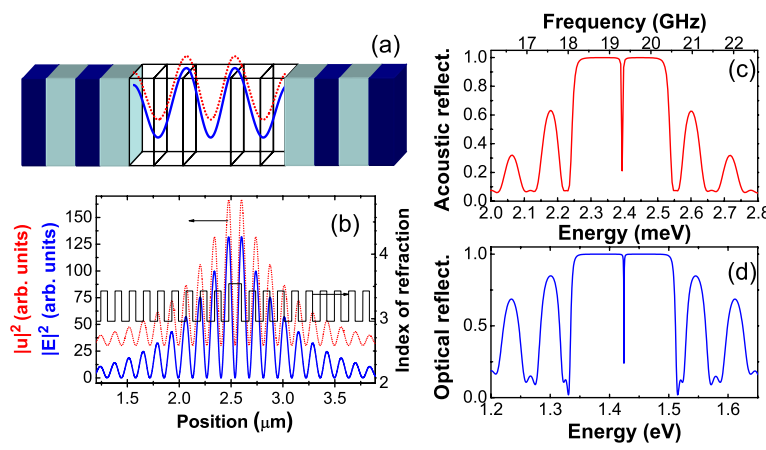

FIG. 1 (color online). (a): Scheme of the GaAs photon-phonon microcavity. Dark regions represent AlGaAs, lighter ones AlAs. The number of layers does not correspond to the real structure. (b) Calculated photon intensity (blue) and phonon square displacement (red dotted curve, vertically shifted) distributions. The central five layers are shown transparent in (a) to display a detail of these curves. The thin lines in (b) represent the material refractive index. (c) and (d): Calculated acoustic and optical reflectivities, respectively. $\left(\lambda_{\text {GaAs }}^{\text {gap }} \sim 870 \mathrm{~nm}\right), \quad d_{\text {cav }} \sim 120 \mathrm{~nm}, \quad$ and thus $g_{\text {om }}$ is expected to assume values in the range $g_{\text {om }} \sim 18 \mathrm{THz} / \mathrm{nm}$.

We study in this Letter the dynamics of the system through a time-resolved experiment in which the mechanical system is prepared in a given state through the impulsive generation of phonons with an ultrafast laser [28]. A delayed probe monitors the change of reflectivity induced by these phonons, which can be modeled as [29,30]

$$
\begin{aligned}
\Delta r(t)= & \frac{i k}{2}\left\{\int_{0}^{L} d z \Delta \epsilon(z, t)\left[E_{\text {probe }}(z)\right]^{2}\right. \\
& \left.+\sum_{m=1}^{2 N}\left(\epsilon_{m-1}-\epsilon_{m}\right) u\left(z_{m}, t\right)\left(a_{m}+b_{m}\right)^{2}\right\} .
\end{aligned}
$$

Here $k$ is the probe light wave number in vacuum, $\epsilon_{m}$ is the dielectric function of layer $m$, and $z_{m}$ is the position of the interface between layers $m$ and $m+1$. The probe field within layer $m$ is $E_{\text {probe }}(z)=a_{m} e^{i \sqrt{\epsilon_{m}} k z}+b_{m} e^{-i \sqrt{\epsilon_{m}} k z}$. $\Delta \epsilon(z, t)$ is the variation in the dielectric function induced by the displacement $u(z, t), \Delta \epsilon(z, t)=P(z) \frac{\partial u(z, t)}{\partial z}$ with $P(z)$ the photoelastic constant. The first term in Eq. (1) is due to the photoelastic effect; i.e., the modulation of the cavity refractive index induced by the phonon strain. The second results from the dynamical change in cavity and DBR thicknesses due to the phonon induced mechanical displacement of the interfaces. The relative importance of each of these terms will be discussed below. As for $\Delta T$ above, the measured differential reflectance $[\Delta R \approx$ $\Re \mathrm{e}(\Delta r(t))$ if absorption is small] can be expressed as $\Delta R=\left(\frac{d R}{d \omega}\right)\left(\frac{d \omega}{d \alpha}\right) \Delta \alpha$. Thus, if $\Delta \alpha$ is known, by determining $\Delta R$ one can obtain $g_{\text {om }}=\frac{d \omega}{d \alpha}$.

Reflection-type pump-probe experiments [28] were performed at room temperature with laser wavelength $\lambda_{\text {laser }}=$ $870 \mathrm{~nm}$ in resonance with the absorption edge of the GaAs spacer. The optomechanic cavity thus involves electronic resonances in the light-matter processes, in addition to the optical and acoustic resonances. Picosecond pulses $(\sim 1 \mathrm{ps,}$ $870 \mathrm{~nm})$ from a mode-locked Ti:sapphire laser were split into the pump (1-300 $\mathrm{mW}$ ) and probe (typically $1 \mathrm{~mW}$ ) pulses [25]. Photon pulses of $1 \mathrm{ps}$ are chosen so that their spectral width matches the finesse of the optical cavity $(\Delta \lambda \sim 0.8 \mathrm{~nm})$. To enhance the impulsive generation of phonons, the laser was tuned in resonance with the optical cavity mode [31]. To maximize the detection sensitivity, as in the $\mathrm{cw}$ optomechanics experiments, the probe laser angle is set so that its energy is tuned to the flank of the optical cavity mode [31]. Note that the laser energy is well within the transparency region of the materials forming the DBRs $\left(\lambda_{\mathrm{AlAs}}^{\mathrm{gap}} \sim 415 \mathrm{~nm}\right.$ and $\left.\lambda_{\mathrm{Al}_{0.18} \mathrm{Ga}_{0.82} \mathrm{As}}^{\mathrm{gap}} \sim 780 \mathrm{~nm}\right)$. Consequently, the phonon impulsive generation occurs mainly at the GaAs cavity spacer. The photoelastic component of the detection is, on the other hand, strongly enhanced at resonance selectively at the GaAs spacer [32].

Figure 2(a) presents a typical $\Delta R$ time trace. The jump at $t \sim 0$ marks the coincidence between pump and probe 
(a)

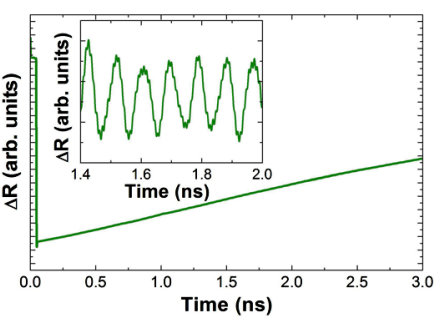

(b)

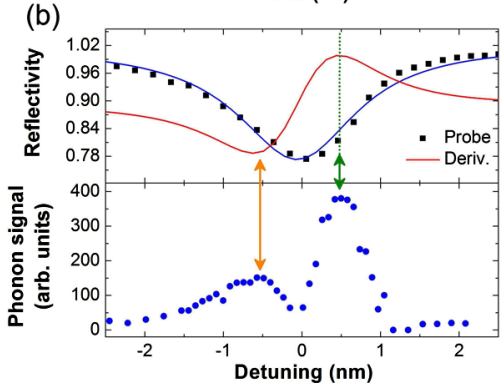

FIG. 2 (color online). (a) Change of reflectivity recorded in the pump-probe experiments. The inset is a detail of the same trace filtered to display only the frequency components $>10 \mathrm{GHz}$. (b) Top panel: Optical reflectivity as a function of the detuning between the laser wavelength and the cavity mode obtained using the probe laser pulses. The full curve is the numerical derivative of the probe reflectivity. Bottom: Coherent phonon peak intensity.

pulses. This signal reflects the variation in the optical cavity reflectivity due to the excitation of electron-hole pairs in the GaAs spacer. The inset is a detail of the same trace filtered to display frequencies $>10 \mathrm{GHz}$ to emphasize the oscillatory part of the signal related to coherent phonons of the structure. Figure 2(b) compares the optical reflectivity obtained using the $\sim 1$ ps probe laser pulses, with the measured coherent phonon peak intensity [derived from the Fourier transform of the signal in (a)] as a function of laser-cavity detuning. Maxima are observed when the probe is tuned to the flanks of the cavity mode [31], unambiguously demonstrating that the observed oscillations are due to the modulation of the cavity reflectivity by phonons belonging to the resonator.

To identify these modes, Fig. 3 (top) displays the Fourier transform amplitude of the measured time trace (integrated for all measured times). Three spectrally narrow peaks are observed at $\nu_{1} \sim 20 \mathrm{GHz}, \quad \nu_{3} \sim 60 \mathrm{GHz}$, and $\nu_{5} \sim$ $100 \mathrm{GHz}$. The width of the modes is determined by the experimental time window ( $0 \leq t \leq 3 \mathrm{~ns}$ ), and it is not reflecting the mode lifetime. Figure 3 (bottom) presents the calculated generated spectral amplitude, considering an impulsive (instantaneous) strain localized at the cavity layer [25,31]. No fitting parameters are used; only nominal thicknesses and published material properties. Three main ultranarrow peaks appear at the same frequencies as the experiment, corresponding to the first three (odd order) acoustic modes confined in the microcavity. The evenorder phonon cavity modes have an odd (respect to the center) strain distribution at the cavity spacer and are thus forbidden by parity (the photon field intensity is even).

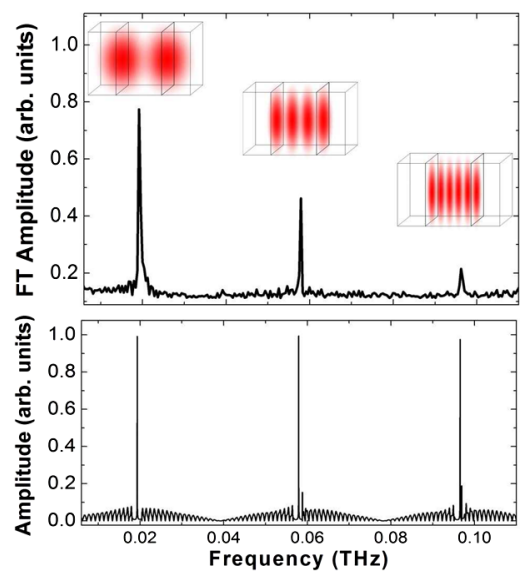

FIG. 3 (color online). Top: Amplitude of the Fourier transform (performed for the full 0-3000-ps experimental time window). The spatial displacements associated with these modes are depicted in the shown insets. Bottom: Calculated generated spectrum.

To track the vibrational dynamics in the optomechanical system, a color map displaying the experimental windowed Fourier transform (WFT) amplitude as a function of time delay for the 20- and 60-GHz modes is shown in Figs. 4(a) and 4(b) [25]. Strong oscillations and beating of the mode amplitudes are observed, together with a strong dip of the $20-\mathrm{GHz}$ mode intensity and a spectral displacement of the $60-\mathrm{GHz}$ mode around 900 ps. Figures 4(c) and 4(d) display the WFT of the differential reflectivity calculated using Eq. (1). The observed behavior results from the interference between the cavity modes and a nonconfined component that escapes the GaAs spacer within the first $\sim 20$ ps, and is echoed back to the GaAs spacer at $\sim 900$ ps [25]. The agreement between experiment and theory supports the use of Eq. (1) to model these cavity optomechanical devices in a simple and clear way.

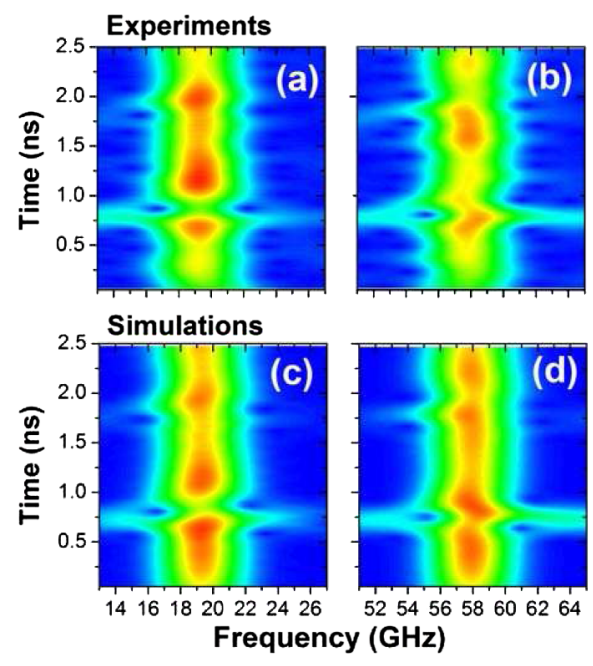

FIG. 4 (color online). (a, b): Detail of the experimental WFT intensity map corresponding to the $20-\mathrm{GHz}$ (a) and $60-\mathrm{GHZ}$ (b) cavity modes. (c, d): Calculated WFT intensity maps. 
We use Eq. (1) to calculate $g_{\mathrm{om}}^{\mathrm{ph}}=\frac{\Delta R}{(d R / d w) \Delta \alpha}$. We emphasize that all required parameters are either experimentally accessible (dielectric and photoelastic constants) or set by design (the cavity structure). To calculate $\Delta R$, wellestablished standard values are used for the dielectric constants of the AlGaAs materials. The photoelastic constants, however, are less known for the different materials and laser energies. We use a value of 200 for the photoelastic constant of GaAs [33]. $\frac{d R}{d \omega}$ is calculated for the probe tuned to the flank of the optical cavity mode using the nominal structure [25]. $\Delta \alpha$ is the displacement associated to the $20-\mathrm{GHz}$ mode [see Fig. 1(b)]. We calculate $g_{\mathrm{om}}^{\mathrm{ph}}=83 \mathrm{THz} / \mathrm{nm}$ for the photoelastic term, and $g_{\mathrm{om}}^{\text {if }}=3.6 \mathrm{THz} / \mathrm{nm}$ for the contribution due to the displacement of spacer and DBR interfaces. We note that values in other NIR optomechanical systems are typically in the $50 \mathrm{MHz} / \mathrm{nm}$ range [34], and up to $\sim 100 \mathrm{GHz} / \mathrm{nm}$ as reported in GaAs disk resonators [10]. A related quantity that describes the length over which a photon's momentum is transferred into the mechanical mode is $L_{\mathrm{om}}=\left(\frac{1}{\omega} \frac{d \omega}{d \alpha}\right)^{-1}$ [22]. We obtain $L_{\mathrm{om}} \sim 600$ or $30 \mathrm{~nm}$ for the pure mechanical or photoelastic contributions, respectively. These values can be compared to several microns for planar Si optomechanical crystals [22]. Nominal $Q$ factors were used in these estimations. However, a discussion on the actual cavity $Q$ factors attainable in these planar DBR-based cavities, and at such high vibrational frequencies, is required. For the reported sample the nominal quality factors are $Q_{\mathrm{op}} \sim Q_{\mathrm{ac}} \sim 10^{3}$, corresponding to cavity escape lifetimes of $\sim 1 \mathrm{ps}$ and $\sim 60 \mathrm{~ns}$, respectively (the difference arising only from the contrasting speeds of light and sound). The theoretical optical $Q$ factor matches the experiment. Phonons are, however, intrinsically anharmonic. From picosecond acoustic studies of $60-\mathrm{GHz}$ phonon in GaAs [35], one can estimate the anharmonic decay lifetime of the $20 \mathrm{GHz}$ first confined mode at $300 \mathrm{~K}$ to be somewhere around $30 \mathrm{~ns}$. Thus, anharmonicity may be slightly limiting the phonon cavity $Q$ factor at $300 \mathrm{~K}$, but not at lower temperatures [35]. Roughness limitations to the phonon lifetime in MBEgrown structures at these frequencies and $Q$ factors are, on the other hand, not expected [36].

With such large values of optomechanical coupling, the question arises whether the proposed system is suitable for the demonstration of stimulated phonon emission through cavity parametric instability $[17,18,20]$. The threshold pump power for a phonon laser based on a two-level optical resonator coupled to a mechanical mode is given by $P_{\text {th }}=\Gamma \gamma^{2} \hbar \omega / \Omega_{R}^{2}$ [17]. Here $\Gamma=$ $\Omega / Q_{\text {mec }}$ and $\gamma=\omega / Q_{\text {opt }}$ are the optical and acoustic mode decay rates, respectively; $\Omega$ is the mechanical frequency and $\Omega_{R}=g_{\text {om }} x_{0} \cdot x_{0}=\sqrt{\hbar / 2 m_{\text {eff }} \Omega_{0}}$ is the zeropoint motion of the mechanical oscillator, and $m_{\mathrm{eff}}$ its effective motional mass. To attain low threshold powers, the resonator should thus have large mechanical and optical $Q$ factors, large optomechanical coupling constant, and small effective mass. As we have shown here, the planar DBR GaAs/AlAs system provides the required optomechanic coupling and large $Q$ factors. Modifying the system by laterally limiting it in a micropillar structure [27] allows us to attain small effective masses. We estimate the motional mass $m_{\mathrm{eff}}$ using the mass density of GaAs and AlAs, assuming that the mode volume is defined by the displacement distribution given in Fig. 1(b) and the micropillar diameter. Considering a micropillar $\lambda / 2$ cavity of $1 \mu \mathrm{m}$ radius and optical and mechanical $Q$ factors $\sim 10^{4}$, we obtain $m_{\text {eff }} \sim 7.7 \mathrm{pg}, \quad x_{0} \sim 5.8 \times 10^{-7} \mathrm{~nm}$ for the $20-\mathrm{GHz}$ mode, $\Omega_{R} \sim 4.8 \times 10^{7} \mathrm{~Hz}$, and thus $P_{\mathrm{th}} \sim$ $60 \mu \mathrm{W}$ ( $P_{\mathrm{th}} \sim 30 \mathrm{~mW}$ if only $g_{\mathrm{om}}^{\text {if }}$ is considered). Different strategies could be implemented to obtain two-level optical resonators based in DBR vertical cavity structures. Namely, angle tuning in large diameter structures [16], vertically coupled double cavities [37], or laterally coupled double pillar structures [38]. The described planar DBR-based $\mathrm{GaAs} / \mathrm{AlAs}$ resonator device naturally connects the domains of cavity optomechanics and optoelectronics. Such pillar structures are standard in the domain of VCSELs, single photon emitters, and polariton condensates. The same cavity optomechanical device operating as a VCSEL could in fact provide the source for the pump photons. In addition, as we have shown, a resonant cavity based on DBRs can be designed so that even with almost perfect mirrors, the transmission in and out of the cavity can be as high as $100 \%$. These two features could be particularly relevant for saser applications.

A. F. acknowledges G. Rozas and A. Bruchhausen for enlightening discussions. The authors acknowledge $\mathrm{V}$. Thierry-Mieg and the late R. Planel for providing the samples used.

[1] C. H. Metzger and K. Karrai, Nature (London) 432, 1002 (2004).

[2] T. Carmon, H. Rokhsari, L. Yang, T. J. Kippenberg, and K. J. Vahala, Phys. Rev. Lett. 94, 223902 (2005).

[3] O. Arcizet, P.-F. Cohadon, T. Briant, M. Pinard, and A. Heidmann, Nature (London) 444, 71 (2006).

[4] J.D. Thompson, B. M. Zwickl, A. M. Jayich, F. Marquardt, S.M. Girvin, and J.G.E. Harris, Nature (London) 452, 72 (2008).

[5] M. S. Kang, A. Nazarkin, A. Brenn, and P. St. J. Russell, Nat. Phys. 5, 276 (2009).

[6] A. Schliesser, O. Arcizet, R. Riviere, and T. J. Kippenberg, Nat. Phys. 5, 509 (2009).

[7] S. Groeblacher, K. Hammerer, M. R. Vanner, and M. Aspelmeyer, Nature (London) 460, 724 (2009).

[8] M. Tomes and T. Carmon, Phys. Rev. Lett. 102, 113601 (2009).

[9] A. H. Safavi-Naeini, T. P. M. Alegre, M. Winger, and O. Painter, Appl. Phys. Lett. 97, 181106 (2010).

[10] L. Ding, C. Baker, P. Senellart, A. Lemaitre, S. Ducci, G. Leo, and I. Favero, Phys. Rev. Lett. 105, 263903 (2010). 
[11] S. Weis, R. Riviere, S. Deleglise, O. Arcizet, E. Gavartin, A. Schliesser, and T. J. Kippenberg, Science 330, 1520 (2010).

[12] J. M. Dobrindt and T. J. Kippenberg, Phys. Rev. Lett. 104, 033901 (2010).

[13] Q. Lin, J. Rosenberg, D. Chang, R. Camacho, M. Eichenfield, K. J. Vahala, and O. Painter, Nat. Photonics 4, 236 (2010).

[14] P. Hu, Phys. Rev. Lett. 44, 417 (1980).

[15] L. G. Tilstra, A. F. M. Arts, and H. W. de Wijn, Phys. Rev. B 76, 024302 (2007).

[16] M. Trigo, A. Bruchhausen, A. Fainstein, B. Jusserand, and V. Thierry-Mieg, Phys. Rev. Lett. 89, 227402 (2002).

[17] I. S. Grudinin, H. Lee, O. Painter, and K. J. Vahala, Phys. Rev. Lett. 104, 083901 (2010).

[18] V. B. Braginsky, S. E. Strygin, and S. E. Vyatchanin, Phys. Lett. A 287, 331 (2001).

[19] C. Zhao, L. Ju, H. Miao, S. Gras, Y. Fan, and D. G. Blair, Phys. Rev. Lett. 102, 243902 (2009).

[20] T. J. Kippenberg, H. Rokhsari, T. Carmon, A. Scherer, and K. J. Vahala, Phys. Rev. Lett. 95, 033901 (2005).

[21] T.J. Kippenberg and K. J. Vahala, Science 321, 1172 (2008).

[22] M. Eichenfield, J. Chan, R. M. Camacho, K. J. Vahala, and O. Painter, Nature (London) 462, 78 (2009).

[23] A. Dousse, J. Suffczynski, A. Beveratos, O. Krebs, A. Lemaître, I. Sagnes, J. Bloch, P. Voisin, and P. Senellart, Nature (London) 466, 217 (2010).

[24] T. Someya, R. Werner, A. Forchel, M. Catalano, R. Cingolani, and Y. Arakawa, Science 285, 1905 (1999).

[25] See Supplemental Material at http://link.aps.org/ supplemental/10.1103/PhysRevLett.110.037403 for [brief description].

[26] R. P. Stanley, R. Houdre, U. Oesterle, M. Ilegems, and C. Weisbuch, Phys. Rev. A 48, 2246 (1993).

[27] V. Loo, L. Lanco, A. Lemaitre, I. Sagnes, O. Krebs, P. Voisin, and P. Senellart, Appl. Phys. Lett. 97, 241110 (2010).
[28] C. Thomsen, H. T. Grahn, H. J. Maris, and J. Tauc, Phys. Rev. B 34, 4129 (1986).

[29] M. F. Pascual-Winter, A. Fainstein, B. Jusserand, B. Perrin, and A. Lemaitre, Phys. Rev. B 85, 235443 (2012).

[30] O. Matsuda, I. Ishii, T. Fukui, J. J. Baumberg, and O. B. Wright, Physica (Amsterdam) B316-317, 205 (2002).

[31] N. D. Lanzillotti-Kimura, A. Fainstein, B. Perrin, and B. Jusserand, Phys. Rev. B 84, 064307 (2011).

[32] A. Bartels, T. Dekorsy, H. Kurz, and K. Kohler, Phys. Rev. Lett. 82, 1044 (1999).

[33] P. Etchegoin, J. Kircher, M. Cardona, C. Grein, and E. Bustarret, Phys. Rev. B 46, 15139 (1992); see also Z. V. Popovic, J. Spitzer, T. Ruf, M. Cardona, R. Notzel, and K. Ploog, Phys. Rev. B 48, 1659 (1993). The reported complex photoelastic constants correspond to the visible green-blue spectral range. We are not aware of similar expressions valid at the $E_{\mathrm{GaAs}}^{\mathrm{gap}}$. The resonant Raman efficiency for scattering by acoustic phonons at the $E_{\mathrm{GaAs}}^{\mathrm{gap}}$ is order-of-magnitude similar to that observed in the greenblue spectral range. We thus use at $E_{\mathrm{GaAs}}^{\mathrm{gap}}$ a similar magnitude for $P(z)$ assuming, however, because of the reduced absorption, an essentially real value.

[34] G. Anetsberger, O. Arcizet, Q. P. Unterreithmeier, R. Riviere, A. Schliesser, E. M. Weig, J.P. Kotthaus, and T. J. Kippenberg, Nat. Phys. 5, 909 (2009).

[35] W. Chen, H. J. Maris, and Z. R. Wasilewski, Philos. Mag. B 70, 687 (1994).

[36] G. Rozas, M.F. Pascual Winter, B. Jusserand, A. Fainstein, B. Perrin, E. Semenova, and A. Lemaître, Phys. Rev. Lett. 102, 015502 (2009).

[37] A. Fainstein, M. Trigo, D. Oliva, B. Jusserand, T. Freixanet, and V. Thierry-Mieg, Phys. Rev. Lett. 86, 3411 (2001).

[38] S. M. de Vasconcellos, A. Calvar, A. Dousse, J. Suffczynski, N. Dupuis, A. Lemaitre, I. Sagnes, J. Bloch, P. Voisin, and P. Senellart, Appl. Phys. Lett. 99, 101103 (2011). 